\title{
Inner Valence Hole Migration in Isopropanol
}

\author{
Oliver Alexander ${ }^{1}$ and the LR25 collaboration, including Thomas Barillot ${ }^{1}$, Bridgette Cooper ${ }^{1,2}$, Taran \\ Driver $^{1,3,4}$, Douglas Garratt ${ }^{1}$, Siqi Li ${ }^{5}$, Agostino Marinelli ${ }^{3,4}$, James P Cryan ${ }^{3,4}$, and Jonathan $\mathbf{P}$ \\ Marangos ${ }^{1}$ et al. \\ 1. Quantum Optics and Laser Science Group, Blackett Laboratory, Imperial College London, London, SW7 2BW, UK. \\ 2. Atomic, Molecular, Optical and Positron Physics Group, University College London, Gower Street, London, WC1E 6BT, UK. \\ 3. Stanford PULSE Institute, SLAC National Accelerator Laboratory, USA. \\ 4. Linac Coherent Light Source, SLAC National Accelerator Laboratory, Menlo Park, California, 94025, USA. \\ 5. Accelerator Research Division, SLAC National Accelerator Laboratory, Menlo Park, California, 94025, USA.
}

Even within the sudden approximation, the removal of one neutral Hartree-Fock orbital does not leave the molecule in a pure one-hole state of the cation; coupling to virtual orbitals leads to a breakdown in the molecular orbital picture. This can be understood as interaction with two-hole one-particle states (singly excited cations) and becomes significant when those states are energetically similar to the one-hole states. This splitting of states is most relevant for inner valence holes which lie above the double ionisation potential. As such, impulsive creation of an inner valence hole (IVH) wavepacket results in a time dependent charge density which is purely electronic in nature (charge migration) [1,2], though it depends on the nuclear geometry at the time of excitation, and subsequent nuclear motion will result in decoherence [3].

We then directly track the relaxation of these holes by probing resonant transient X-ray absorption detected via now opened Auger decay channels from a second X-ray pulse, the 'probe', which is resonant between the oxygen $1 \mathrm{~s}$ and hole state of interest $(514 \mathrm{eV})$. We made these measurements with pump-probe pulses of $7 \mathrm{fs}$ and 2.5 fs FWHM, in two separate measurements, at the linac coherent light source (LCLS) free electron laser. Unlike measurements which utilise near-infrared pulses to create hole wavepackets via strong field ionization, the use of all X-ray pulses weakly perturbs the system and allows us to study the field free charge dynamics. By measuring the change in resonant Auger yield at short time delays we are able to identify a transient signal dependant on the probe energy, which naturally fluctuates due to the self amplified spontaneous emission mode used (Fig. 1a). From this, we identify, from their energetics, resonances corresponding to ionization of the $6 a$ and $7 a$ molecular orbitals (Fig. 1b and Fig. 1c respectively). The corresponding delay dependence identifies the relaxation of the $6 a$ and $7 a$ IVHs upon impulsive excitation.
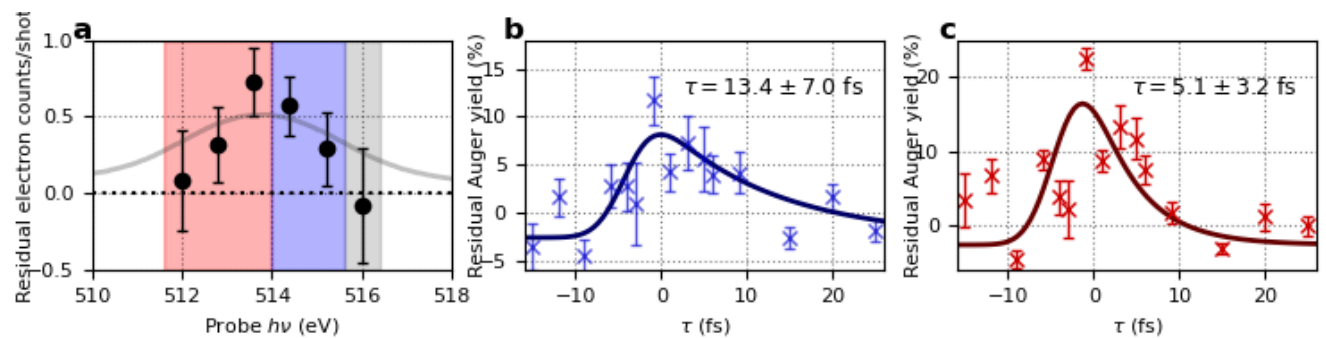

Fig. 1 a Probe central photon energy dependence of residual signal at delay $\sim 0 \mathrm{fs}$.b Time dependence of residual resonant high kinetic energy electron signal at higher probe photon energy, about expected $1 a \rightarrow 7 a$ transition (blue), and c time dependence of residual resonant high energy electron yield at lower probe photon energy about expected $1 a \rightarrow 6 a$ transition (red). Fits to an exponentially modified Gaussian functional form are shown in black and the extracted decay time is given in each panel.

We measure a lifetime of the $6 a$ orbital hole survival probability of $5.1 \pm 3.2 \mathrm{fs}$ ( $7 \mathrm{fs}$ pulse measurement) and $1 \pm 2 \mathrm{fs}$ ( $2.5 \mathrm{fs}$ pulse measurement), with both values consistent with our theoretical calculations performed within the $\mathrm{ADC}(2) \mathrm{x}$ framework which shows a decay of lifetime $2.3 \mathrm{fs}$. For the $7 a$ orbital, the measured hole decay time was $13.4 \pm 7.0 \mathrm{fs}$ a value in good agreement with an auxiliary Coulomb explosion VUV/IR measurement of $13 \mathrm{fs}$. Our measurements present the first observation of the temporal signature of frustrated Auger-Meitner transitions, as well as strong evidence of the influence of nuclear motion on the longer timescale of the $7 a$ orbital. The method employed here will be applicable to systems with sufficient size for the electronic wave packet to migrate across multiple atomic sites and be tracked via X-ray probes covering appropriate resonances. Probing charge migration and subsequent photochemical activity using X-ray pump-probe methodology with sub-femtosecond pulses will deepen our understanding of the first moments following the photo-ionization of matter.

\section{References}

[1] L. S. Cederbaum and J. Zobeley, "Ultrafast charge migration by electron correlation," Chem. Phys. Lett., 307 (3-4), 205-210 (1999). [2] J. Breidbach and L. S. Cederbaum, "Universal attosecond response to the removal of an electron," Phys. Rev. Lett., 94.3, 033901 (2005) [3] A. J. Jenkins, M. Vacher, R. M. Twidale, M. J Bearpark and M. A. Robb, "Charge migration in polycyclic norbornadiene cations: Winning the race against decoherence," J. Chem. Phys., 145.16, 164103 (2016). 